
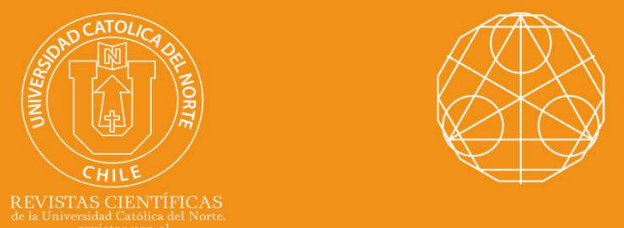

\title{
Stability of a general $p$-radical functional equation related to additive mappings in 2-Banach spaces
}

Sadeq A. A. AL-Ali ${ }^{1}$

Muaadh Almahalebi² ${ }^{2}$ orcid.org/0000-0003-0647-8286

Youssfi Elkettani ${ }^{3}$ (10 orcid.org/0000-0002-2658-4687

Ibn Tofaïl University, Dept. of Mathematics, Faculty of Sciences, Kenitra, Morocco.

1@ sadeqalali2018@gmail.com ; ${ }^{2}$ muaadh1979@hotmail.fr ;

3匹 elkettani@ui-ibntofail.ac.ma

Received: December 2019 | Accepted: October 2020

\section{Abstract:}

In this paper, we introduce and solve a new general p-radical functional equation

$$
f\left(\sqrt[p]{\sum_{i=1}^{k} x_{i}^{p}}\right)=\sum_{i=1}^{k} f\left(x_{i}\right), \quad k, p \in \mathbf{N}_{2} .
$$

Also, we investigate some stability and hyperstability results for the considered equation in 2-Banach spaces. In addition, we prove the hyperstability of the inhomogeneous p-radical functional equation

$$
f\left(\sqrt[p]{\sum_{i=1}^{k} x_{i}^{p}}\right)=\sum_{i=1}^{k} f\left(x_{i}\right)+G\left(x_{1}, x_{2}, \ldots, x_{k}\right) .
$$

Keywords: Stability; Hyperstability; 2-normed space; Fixed point method; Radical functional equations.

MSC (2020): 39B82, 39B62, 47H14, 47J20, 47H10.

\section{Cite this article as (IEEE citation style):}

S. A. A. AL-Ali, M. Almahalebi, and Y. Elkettani, "Stability of a general $p$-radical functional equation related to additive mappings in 2-Banach spaces", Proyecciones (Antofagasta, On line), vol. 40, no. 1, pp. 49-71, 2021, doi: 10.22199/issn.07176279-2021-01-0004

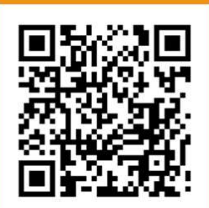

Article copyright: (C) 2021 Sadeq A. A. AL-Ali, Muaadh Almahalebi, and Youssfi Elkettani. This is an open access article distributed under the terms of the Creative Commons License, which permits unrestricted use and distribution provided the original author and source are credited. 


\section{Introduction}

Throughout this paper, we will denote the set of natural numbers by $\mathbf{N}$, the set of real numbers by $\mathbf{R}$ and $\mathbf{R}_{+}=[0, \infty)$ the set of nonnegative real numbers. By $\mathbf{N}_{m}, m \in \mathbf{N}$, we will denote the set of all natural numbers greater than or equal to $m$.

The notion of linear 2-normed spaces was introduced by Gähler [16],[17] in the middle of 1960s. We need to recall some basic facts concerning 2- normed spaces and some preliminary results.

Definition 1.1. Let $X$ be a real linear space with $\operatorname{dim} X>1$ and $\|.,$.$\| :$ $X \times X \longrightarrow[0, \infty)$ be a function satisfying the following properties:

1. $\|x, y\|=0$ if and only if $x$ and $y$ are linearly dependent,

2. $\|x, y\|=\|y, x\|$,

3. $\|\lambda x, y\|=|\lambda|\|x, y\|$,

4. $\|x, y+z\| \leq\|x, y\|+\|x, z\|$,

for all $x, y, z \in X$ and $\lambda \in \mathbf{R}$. Then the function $\|.,$.$\| is called a 2-norm$ on $X$ and the pair $(X,\|.,\|$.$) is called a linear 2-normed space. Sometimes$ the condition (4) called the triangle inequality.

Example 1.2. For $x=\left(x_{1}, x_{2}\right), y=\left(y_{1}, y_{2}\right) \in X=\mathbf{R}^{2}$, the Euclidean 2-norm $\|x, y\|_{\mathbf{R}^{2}}$ is defined by

$$
\|x, y\|_{\mathbf{R}^{2}}=\left|x_{1} y_{2}-x_{2} y_{1}\right| .
$$

Lemma 1.3. Let $(X,\|.,\|$.$) be a 2-normed space. If x \in X$ and $\|x, y\|=0$, for all $y \in X$, then $x=0$.

Definition 1.4. A sequence $\left\{x_{k}\right\}$ in a 2-normed space $X$ is called a convergent sequence if there is an $x \in X$ such that

$$
\lim _{k \rightarrow \infty}\left\|x_{k}-x, y\right\|=0
$$

for all $y \in X$. If $\left\{x_{k}\right\}$ converges to $x$, write $x_{k} \longrightarrow x$ with $k \longrightarrow \infty$ and call $x$ the limit of $\left\{x_{k}\right\}$. In this case, we also write $\lim _{k \rightarrow \infty} x_{k}=x$. 
Definition 1.5. A sequence $\left\{x_{k}\right\}$ in a 2-normed space $X$ is said to be a Cauchy sequence with respect to the 2-norm if

$$
\lim _{k, l \rightarrow \infty}\left\|x_{k}-x_{l}, y\right\|=0,
$$

for all $y \in X$. If every Cauchy sequence in $X$ converges to some $x \in X$, then $X$ is said to be complete with respect to the 2-norm. Any complete 2-normed space is said to be a 2-Banach space.

Now, we state the following results as lemma (See [22] for the details).

Lemma 1.6. Let $X$ be a 2-normed space. Then,

1. $|\|x, z\|-\|y, z\|| \leq\|x-y, z\|$ for all $x, y, z \in X$,

2. if $\|x, z\|=0$ for all $z \in X$, then $x=0$,

3. for a convergent sequence $x_{n}$ in $X$,

$$
\lim _{n \longrightarrow \infty}\left\|x_{n}, z\right\|=\left\|\lim _{n \longrightarrow \infty} x_{n}, z\right\|
$$

for all $z \in X$.

The basic notion of stability for a functional equation emerges when defining, in some way, the class of approximate solutions of the given functional equation, one can ask whether each mapping from this class can be somehow approximated by an exact solution of the considered equation. In other words, when one replaces a functional equation by an inequality which acts as a perturbation of the considered equation.

The study of stability problems of functional equations was motivated by a question of S. M. Ulam [26] asked in 1940.

\section{Ulam's problem:}

Let $\left(G_{1}, *_{1}\right)$ be a group and let $\left(G_{2}, *_{2}\right)$ be a metric group with a metric $d(.,$.$) . Given \varepsilon>0$, does there exists a $\delta>0$ such that if a mapping $h: G_{1} \rightarrow G_{2}$ satisfies the inequality

$$
d\left(h\left(x *_{1} y\right), h(x) *_{2} h(y)\right)<\delta
$$

for all $x, y \in G_{1}$, then there exists a homomorphism $H: G_{1} \rightarrow G_{2}$ with

$$
d(h(x), H(x))<\varepsilon
$$


for all $x \in G_{1}$ ?

Since then, this question has attracted the attention of many researchers. The first result giving a partial answer to this question is due to D. H. Hyers [19]. Subsequently, this result was extended and generalized in several ways. The following theorem is the most classical result concerning the Hyers-Ulam stability:

Theorem 1.7. Let $X$ and $Y$ be two normed spaces, $Y$ be complete, $c \geq 0$ and $p=1$ be a real number. Let $f: X \rightarrow Y$ be an operator such that

$$
\|f(x+y)-f(x)-f(y)\| \leq c\left(\|x\|^{p}+\|y\|^{p}\right), \quad x, y \in X \backslash\{0\} .
$$

Then there exists an additive mapping $T: X \rightarrow Y$ with

$$
\|f(x)-T(x)\| \leq \frac{c}{\left|1-2^{p-1}\right|}\|x\|^{p}, \quad x \in X \backslash\{0\} .
$$

It is due to T. Aoki [5] (for $0<p<1$ ), Z. Gajda [18] (for $p>1$ ) and Th. M. Rassias [25] (for $p<0$ ). Moreover, an example is given in [18] from which it follows that analogous result for $p=1$ is not true. For $p$ $=0$ it is the first result of stability proved by Hyers [19]. Another generalization of the result of Hyers was considered by J. M. Rassias who has proved the following theorem:

Theorem 1.8. [23]-[24] Let $X$ and $Y$ be two normed spaces, $Y$ be complete, $c \geq 0$ and $r, s$ be a real numbers such that $q=r+s=1$. Let $f: X \rightarrow Y$ be an operator such that

$$
\|f(x+y)-f(x)-f(y)\| \leq c\|x\|^{r}\|y\|^{s}, \quad x, y \in X \backslash\{0\} .
$$

Then there exists an additive mapping $T: X \rightarrow Y$ with

$$
\|f(x)-T(x)\| \leq \frac{c}{\left|2^{q}-2\right|}\|x\|^{q}, \quad x \in X \backslash\{0\} .
$$

Now, it is known that for $p<0$ we have the hyperstability result, that is $f$ satisfying (1.1) must be additive (see [9]). Also, J. Brzdęk [6] showed that estimation (1.2) is optimal for $p \geq 0$ in the general case. 
Recently, Brzdęk [9] showed that Theorem 1.7 can be significantly improved; namely, in the case $p<0$, each $f: E_{1} \rightarrow E_{2}$ satisfying (1.1) must actually be additive, and the assumption of completeness of $E_{2}$ is not necessary. It is regrettable that this result does not remain valid if we restrict the domain of $f$ (see the further detail in [11]). But then again, several mathematicians showed that the fixed point method is an another very efficient and convenient tool for proving the Hyers-Ulam stability for a quite wide class of functional equations (see [10]). Brzdęk et al. [7] proved the fixed point theorem for a nonlinear operator in metric spaces and used this result to study the Hyers-Ulam stability of some functional equations in non-Archimedean metric spaces. In this work, they also obtained the fixed point result in arbitrary metric spaces as follows:

Theorem 1.9. [7] Let $X$ be a nonempty set, $(Y, d)$ be a complete metric space, and $\Lambda: Y^{X} \rightarrow Y^{X}$ be a non-decreasing operator satisfying the hypothesis

$$
\lim _{n \rightarrow \infty} \Lambda \delta_{n}=0
$$

for every sequence $\left\{\delta_{n}\right\}_{n \in \mathbf{N}}$ in $Y^{X}$ with

$$
\lim _{n \rightarrow \infty} \delta_{n}=0 .
$$

Suppose that $\mathcal{T}: Y^{X} \rightarrow Y^{X}$ is an operator satisfying the inequality

$$
d(\mathcal{T} \xi(x), \mathcal{T} \mu(x)) \leq \Lambda(\Delta(\xi, \mu))(x), \quad \xi, \mu \in Y^{X}, x \in X,
$$

where $\Delta: Y^{X} \times Y^{X} \rightarrow \mathbf{R}_{+}^{X}$ is a mapping which is defined by

$$
\Delta(\xi, \mu)(x):=d(\xi(x), \mu(x)) \quad \xi, \mu \in Y^{X}, x \in X .
$$

If there exist functions $\varepsilon: X \rightarrow \mathbf{R}_{+}$and $\varphi: X \rightarrow Y$ such that

$$
d((\mathcal{T} \varphi)(x), \varphi(x)) \leq \varepsilon(x)
$$

and

$$
\varepsilon^{*}(x):=\sum_{n \in \mathbf{N}_{0}}\left(\Lambda^{n} \varepsilon\right)(x)<\infty
$$

for all $x \in X$, then the limit 


$$
\lim _{n \rightarrow \infty}\left(\left(\mathcal{T}^{n} \varphi\right)\right)(x)
$$

exists for each $x \in X$. Moreover, the function $\psi \in Y^{X}$ defined by

$$
\psi(x):=\lim _{n \rightarrow \infty}\left(\left(\mathcal{T}^{n} \varphi\right)\right)(x)
$$

is a fixed point of $\mathcal{T}$ with

$$
d(\varphi(x), \psi(x)) \leq \varepsilon^{*}(x)
$$

for all $x \in X$.

In 2013, Brzdȩk [8] gave the fixed point result by applying Theorem 1.9 as follows:

Theorem 1.10. [8] Let $X$ be a nonempty set, $(Y, d)$ be a complete metric space, $f_{1}, \ldots, f_{r}: X \rightarrow X$ and $L_{1}, \ldots, L_{r}: X \rightarrow \mathbf{R}_{+}$be given mappings. Suppose that $\mathcal{T}: Y^{X} \rightarrow Y^{X}$ and $\Lambda: \mathbf{R}_{+}^{X} \rightarrow \mathbf{R}_{+}^{X}$ are two operators satisfying the conditions

$$
d(\mathcal{T} \xi(x), \mathcal{T} \mu(x)) \leq \sum_{i=1}^{r} L_{i}(x) d\left(\xi\left(f_{i}(x)\right), \mu\left(f_{i}(x)\right)\right)
$$

for all $\xi, \mu \in Y^{X}, x \in X$ and

$$
\Lambda \delta(x):=\sum_{i=1}^{r} L_{i}(x) \delta\left(f_{i}(x)\right), \quad \delta \in \mathbf{R}_{+}^{X}, x \in X .
$$

If there exist functions $\varepsilon: X \rightarrow \mathbf{R}_{+}$and $\varphi: X \rightarrow Y$ such that

$$
d(\mathcal{T} \varphi(x), \varphi(x)) \leq \varepsilon(x)
$$

and

$$
\varepsilon^{*}(x):=\sum_{n=0}^{\infty}\left(\Lambda^{n} \varepsilon\right)(x)<\infty
$$

for all $x \in X$, then the limit (1.9) exists for each $x \in X$. Moreover, the function (1.10) is a fixed point of $\mathcal{T}$ with (1.11) for all $x \in X$. 
Then by using this theorem, Brzdȩk [8] improved, extended and complemented several earlier classical stability results concerning the additive Cauchy equation (in particular Theorem 1.7). Over the last few years, many mathematicians have investigated various generalizations, extensions and applications of the Hyers-Ulam stability of a number of functional equations (see, for instance, [10], [11] and references therein), in particular, the stability problem of the radical functional equations in various spaces was proved in $[1,2,3,14,15,20,21]$.

In 2019, M. Almahalebi et al. [4] stated and proved an analogue of Theorem 1.10 in 2-Banach spaces as follows:

Theorem 1.11. [4] Let $X$ be a nonempty set, $(Y,\|\cdot, \cdot\|)$ be a 2-Banach space, $g: X \rightarrow Y$ be a surjective mapping and let $f_{1}, \ldots, f_{r}: X \rightarrow X$ and $L_{1}, \ldots, L_{r}: X \rightarrow \mathbf{R}_{+}$be given mappings. Suppose that $\mathcal{T}: Y^{X} \rightarrow Y^{X}$ and $\Lambda: \mathbf{R}_{+}^{X \times X} \rightarrow \mathbf{R}_{+}^{X \times X}$ are two operators satisfying the conditions

$$
\|\mathcal{T} \xi(x)-\mathcal{T} \mu(x), g(z)\| \leq \sum_{i=1}^{r} L_{i}(x)\left\|\xi\left(f_{i}(x)\right)-\mu\left(f_{i}(x)\right), g(z)\right\|
$$

for all $\xi, \mu \in Y^{X}, x, z \in X$ and

$$
\Lambda \delta(x, z):=\sum_{i=1}^{r} L_{i}(x) \delta\left(f_{i}(x), z\right), \quad \delta \in \mathbf{R}_{+}^{X \times X}, x, z \in X .
$$

If there exist functions $\varepsilon: X \times X \rightarrow \mathbf{R}_{+}$and $\varphi: X \rightarrow Y$ such that

$$
\|\mathcal{T} \varphi(x)-\varphi(x), g(z)\| \leq \varepsilon(x, z)
$$

and

$$
\varepsilon^{*}(x, z):=\sum_{n=0}^{\infty}\left(\Lambda^{n} \varepsilon\right)(x, z)<\infty
$$

for all $x, z \in X$, then the limit

$$
\lim _{n \rightarrow \infty}\left(\left(\mathcal{T}^{n} \varphi\right)\right)(x)
$$

exists for each $x \in X$. Moreover, the function $\psi: X \rightarrow Y$ defined by

$$
\psi(x):=\lim _{n \rightarrow \infty}\left(\left(\mathcal{T}^{n} \varphi\right)\right)(x)
$$


is a fixed point of $\mathcal{T}$ with

$$
\|\varphi(x)-\psi(x), g(z)\| \leq \varepsilon^{*}(x, z)
$$

for all $x, z \in X$.

This theorem is the main tool to prove our stability results. Another version of Theorem 1.11 in 2-Banach space can be found in [13].

In this paper, we achieve the general solutions of the following general $p$-radical functional equation:

$$
f\left(\sqrt[p]{\sum_{i=1}^{k} x_{i}^{p}}\right)=\sum_{i=1}^{k} f\left(x_{i}\right), \quad k, p \in \mathbf{N}_{2}
$$

related to additive mapping and discuss the Hyers-Ulam stability problem in 2-Banach spaces by using Brzdȩks fixed point result. Also, we investigate some hyperstability results for the considered equation.

\section{Solution of the equation (1.23)}

In this section, we give the general solution of functional equation (1.23). The proof of the following theorem has been patterned on the reasoning in [12].

Theorem 2.1. Let $Y$ be a linear space. A function $f: \mathbf{R} \rightarrow Y$ satisfies the functional equation (1.23) if and only if

$$
f(x)=F\left(x^{p}\right), \quad x \in \mathbf{R},
$$

with some additive function $F: \mathbf{R} \rightarrow Y$.

Proof. Indeed, It is not hard to check without any problem that if $f: \mathbf{R} \rightarrow Y$ satisfies (2.1), then it is a solution to (1.23). On the other hand, if $f: \mathbf{R} \rightarrow Y$ is a solution of (1.23), then we consider the following two cases:

Case 1: $p$ is even:

We write $F_{0}(x)=f(\sqrt[p]{x})$, for $x \in[0,+\infty)$, then from (1.23) we obtain that

$$
F_{0}\left(\sum_{i=1}^{k} x_{i}\right)=\sum_{i=1}^{k} F_{0}\left(x_{i}\right)
$$


for all $x_{i} \in[0,+\infty)$ with $i=1,2, \ldots, k$. Letting $x_{1}=x$ and $x_{i}=0$ for $i=$ $2,3, \ldots, k$ in $(1.23)$, we obtain that $f$ is even. So, $f(-x)=f(x)=F_{0}\left(x^{p}\right)$, for all $x \in[0,+\infty)$. Now, it is enough to observe that there is an additive $F: \mathbf{R} \rightarrow Y$ with $F(x)=F_{0}(x)$ for all $x \in[0,+\infty)$.

Case 2: $p$ is odd:

By similar method in case 1 , we can write $F(x)=f(\sqrt[p]{x})$, for all $x \in \mathbf{R}$. Then, we get that there exists an additive $F: \mathbf{R} \rightarrow Y$ with $F(x)=F_{0}(x)$ for all $x \in \mathbf{R}$. This completes the proof.

\section{Approximation of the $p$-radical functional equation (1.23)}

In the following two theorems, we use Theorem 1.11 to investigate the generalized Hyers-Ulam stability of the $p$-radical functional equation (1.23) in 2-Banach spaces. Hereafter, we assume that $(Y,\|\cdot, \cdot\|)$ is a 2-Banach space.

Theorem 3.1. Let $h_{i}: \mathbf{R} \times \mathbf{R} \rightarrow \mathbf{R}_{+}$be functions such that

$\mathcal{U}:=\left\{n \in \mathbf{N}: \alpha_{n}:=\prod_{i=1}^{k} \lambda_{i}\left((k-1) n^{p}+1\right)+(k-1) \prod_{i=1}^{k} \lambda_{i}\left(n^{p}\right)<1\right\} \neq \phi$,

where

$$
\lambda_{i}(n):=\inf \left\{t \in \mathbf{R}_{+}: h_{i}\left(n x^{p}, z\right) \leq t h_{i}\left(x^{p}, z\right), \quad x, z \in \mathbf{R}\right\}
$$

for all $n \in \mathbf{N}$, with $i=1,2, \ldots, k$. Assume that $f: \mathbf{R} \rightarrow Y$ satisfies the inequality

$$
\left\|f\left(\sqrt[p]{\sum_{i=1}^{k} x_{i}^{p}}\right)-\sum_{i=1}^{k} f\left(x_{i}\right), g(z)\right\| \leq \prod_{i=1}^{k} h_{i}\left(x^{p}, z\right)
$$

for all $x_{i}, z \in \mathbf{R}$, with $i=1,2, \ldots, k$, where $g: X \rightarrow Y$ be a surjective mapping. Then there exists a unique $p$-radical function $F: \mathbf{R} \rightarrow Y$ such that

$$
\|f(x)-F(x), g(z)\| \leq \lambda_{0} \prod_{i=1}^{k} h_{i}\left(x^{p}, z\right)
$$


for all $x, z \in \mathbf{R}$, where

$$
\lambda_{0}:=\inf _{n \in \mathcal{U}}\left\{\frac{\prod_{i=1}^{k-1} \lambda_{i}\left(n^{p}\right)}{1-\prod_{i=1}^{k} \lambda_{i}\left((k-1) n^{p}+1\right)-(k-1) \prod_{i=1}^{k} \lambda_{i}\left(n^{p}\right)}\right\} .
$$

Proof. $\quad$ For $i=1,2, \ldots,(k-1)$, replacing $x_{i}$ with $m x$ and $x_{k}$ with $x$, where $x_{i} \in \mathbf{R}$ and $m \in \mathbf{N}$, in inequality (3.3), we get

$$
\left\|f\left(\sqrt[p]{\left((k-1) m^{p}+1\right) x^{p}}\right)-(k-1) f(m x)-f(x), g(z)\right\| \leq h_{k}\left(x^{p}, z\right) \prod_{i=1}^{k-1} h_{i}\left(m^{p} x^{p}, z\right)
$$

for all $x, z \in \mathbf{R}$. For each $m \in \mathbf{N}$, we define the operator $\mathcal{T}_{m}: Y^{\mathbf{R}} \rightarrow Y^{\mathbf{R}}$ by

$(3.6) \mathcal{T}_{m} \xi(x):=\xi\left(\sqrt[p]{\left((k-1) m^{p}+1\right) x^{p}}\right)-(k-1) \xi(m x), \quad \xi \in Y^{\mathbf{R}}, x \in \mathbf{R}$.

Further put

$$
\varepsilon_{m}(x, z):=h_{k}\left(x^{p}, z\right) \prod_{i=1}^{k-1} h_{i}\left(m^{p} x^{p}, z\right), \quad x, z \in \mathbf{R}
$$

and observe that

$\varepsilon_{m}(x, z)=h_{k}\left(x^{p}, z\right) \prod_{i=1}^{k-1} h_{i}\left(m^{p} x^{p}, z\right) \leq \prod_{i=1}^{k-1} \lambda_{i}\left(m^{p}\right) \prod_{i=1}^{k} h_{i}\left(x^{p}, z\right), \quad x, z \in \mathbf{R}, m \in \mathbf{N}$.

Then the inequality (3.5) takes the form

$$
\left\|\mathcal{T}_{m} f(x)-f(x), g(z)\right\| \leq \varepsilon_{m}(x, z), \quad x, z \in \mathbf{R} .
$$

Furthermore, for every $x, z \in \mathbf{R}, \xi, \mu \in Y^{\mathbf{R}}$, we obtain

$$
\begin{aligned}
\left\|\mathcal{T}_{m} \xi(x)-\mathcal{T}_{m} \mu(x), g(z)\right\|= & \| \xi\left(\sqrt[p]{\left((k-1) m^{p}+1\right) x^{p}}\right)-(k-1) \xi(m x) \\
& -\mu\left(\sqrt[p]{\left((k-1) m^{p}+1\right) x^{p}}\right)+(k-1) \mu(m x), g(z) \| \\
\leq & \left\|(\xi-\mu)\left(\sqrt[p]{\left((k-1) m^{p}+1\right) x^{p}}\right), g(z)\right\| \\
& +(k-1)\|(\xi-\mu)(m x), g(z)\|
\end{aligned}
$$


This brings us to define the operator $\Lambda_{m}: \mathbf{R}_{+}^{\mathbf{R} \times \mathbf{R}} \rightarrow \mathbf{R}_{+}^{\mathbf{R} \times \mathbf{R}}$ by $\Lambda_{m} \delta(x, z):=\delta\left(\sqrt[p]{\left((k-1) m^{p}+1\right) x^{p}}, z\right)+(k-1) \delta(m x, z), \quad \delta \in \mathbf{R}_{+}^{\mathbf{R} \times \mathbf{R}}, x, z \in \mathbf{R}$.

For each $m \in \mathbf{N}$, the above operator has the form described in (1.17) with $f_{1}(x)=\sqrt[p]{\left((k-1) m^{p}+1\right) x^{p}}, f_{2}(x)=m x$ and $L_{1}(x)=1, L_{2}(x)=$ $k-1$ for all $x \in \mathbf{R}$. By induction, we will show that for each $x, z \in \mathbf{R}$, $n \in \mathbf{N}_{0}$, and $m \in \mathcal{U}$ we have

$$
\Lambda_{m}^{n} \varepsilon_{m}(x, z) \leq \prod_{i=1}^{k-1} \lambda_{i}\left(m^{p}\right) \alpha_{m}^{n} \prod_{i=1}^{k} h_{i}\left(x^{p}, z\right),
$$

where $\alpha_{m}^{n}$ is given by (3.1). From (3.7) and (3.8), we obtain that the inequality (3.11) holds for $n=0$. Next, we will assume that (3.11) holds for $n=r$, where $r \in \mathbf{N}$. Then we have

$$
\begin{aligned}
\left(\Lambda_{m}^{r+1} \varepsilon_{m}\right)(x, z)= & \Lambda_{m}\left(\left(\Lambda_{m}^{r} \varepsilon_{m}\right)(x, z)\right) \\
= & \left(\Lambda_{m}^{r} \varepsilon_{m}\right)\left(\sqrt[p]{\left.\left((k-1) m^{p}+1\right) x^{p}, z\right)}\right. \\
& +(k-1)\left(\Lambda_{m}^{r} \varepsilon_{m}\right)(m x, z) \\
\leq & \prod_{i=1}^{k-1} \lambda_{i}\left(m^{p}\right) \alpha_{m}^{r} \prod_{i=1}^{k} h_{i}\left(\left((k-1) m^{p}+1\right) x^{p}, z\right) \\
& +(k-1) \prod_{i=1}^{k-1} \lambda_{i}\left(m^{p}\right) \alpha_{m}^{r} \prod_{i=1}^{k} h_{i}\left(m^{p} x^{p}, z\right) \\
\leq & \prod_{i=1}^{k-1} \lambda_{i}\left(m^{p}\right) \alpha_{m}^{r} \prod_{i=1}^{k} h_{i}\left(x^{p}, z\right)\left(\prod_{i=1}^{k} \lambda_{i}\left((k-1) m^{p}+1\right)\right. \\
& +(k-1) \prod_{i=1}^{k} \lambda_{i}\left(m^{p}\right) \\
= & \prod_{i=1}^{k-1} \lambda_{i}\left(m^{p}\right) \alpha_{m}^{r+1} \prod_{i=1}^{k} h_{i}\left(x^{p}, z\right)
\end{aligned}
$$

for all $x, z \in \mathbf{R}, m \in \mathcal{U}$. This shows that (3.11) holds for $n=r+1$. Now we can conclude that the inequality (3.11) holds for all $n \in \mathbf{N}_{0}$. Hence, we obtain

$$
\begin{aligned}
\varepsilon_{m}^{*}(x, z) & =\sum_{n=0}^{\infty}\left(\Lambda_{m}^{n} \varepsilon_{m}\right)(x, z) \\
& \leq \sum_{n=0}^{\infty}\left(\prod_{i=1}^{k-1} \lambda_{i}\left(m^{p}\right) \alpha_{m}^{n} \prod_{i=1}^{k} h_{i}\left(x^{p}, z\right)\right) \\
& =\frac{\prod_{i=1}^{k-1} \lambda_{i}\left(m^{p}\right) \prod_{i=1}^{k} h_{i}\left(x^{p}, z\right)}{1-\alpha_{m}}<\infty
\end{aligned}
$$

for all $x, z \in \mathbf{R}, m \in \mathcal{U}$. Therefore, according to Theorem 1.11 with $\varphi=f$ and $X=\mathbf{R}$, we get that the limit

$$
F_{m}(x):=\lim _{n \rightarrow \infty}\left(\mathcal{T}_{m}^{n} f\right)(x)
$$


exists for each $x, z \in \mathbf{R}$ and $m \in \mathcal{U}$, and

$$
\left\|f(x)-F_{m}(x), g(z)\right\| \leq \frac{\prod_{i=1}^{k-1} \lambda_{i}\left(m^{p}\right) \prod_{i=1}^{k} h_{i}\left(x^{p}, z\right)}{1-\alpha_{m}}, x, z \in \mathbf{R}, m \in \mathcal{U}
$$

To prove that $F_{m}$ satisfies the functional equation (1.23), just prove the following inequality

$$
\left\|\mathcal{T}_{m}^{n} f\left(\sqrt[p]{\sum_{i=1}^{k} x_{i}^{p}}\right)-\sum_{i=1}^{k} \mathcal{T}_{m}^{n} f\left(x_{i}\right), g(z)\right\|_{*} \leq \alpha_{m}^{n} \prod_{i=1}^{k} h_{i}\left(x^{p}, z\right)
$$

for every $x_{i}, z \in \mathbf{R}, n \in \mathbf{N}_{0}$, and $m \in \mathcal{U}$. Since the case $n=0$ is just (3.3), take $r \in \mathbf{N}$ and assume that (3.13) holds for $n=r$ and every $x_{i}, z \in \mathbf{R}$, $m \in \mathcal{U}$. Then, for each $x_{i}, z \in \mathbf{R}$ and $m \in \mathcal{U}$, we get

$$
\begin{aligned}
& \left\|\mathcal{T}_{m}^{r+1} f\left(\sqrt[p]{\sum_{i=1}^{k} x_{i}^{p}}\right)-\sum_{i=1}^{k} \mathcal{T}_{m}^{r+1} f\left(x_{i}\right), g(z)\right\| \\
& =\| \mathcal{T}_{m}^{r} f\left(\sqrt[p]{\left((k-1) m^{p}+1\right) \sum_{i=1}^{k} x_{i}^{p}}\right) \\
& -(k-1) \mathcal{T}_{m}^{r} f\left(m \sqrt[p]{\sum_{i=1}^{k} x_{i}^{p}}\right)-\sum_{i=1}^{k} \mathcal{T}_{m}^{r} f\left(\sqrt[p]{\left((k-1) m^{p}+1\right) x_{i}^{p}}\right) \\
& +(k-1) \sum_{i=1}^{k} \mathcal{T}_{m}^{r} f\left(m x_{i}\right), g(z) \\
& \leq \| \mathcal{T}_{m}^{r} f\left(\sqrt[p]{\left((k-1) m^{p}+1\right) \sum_{i=1}^{k} x_{i}^{p}}\right) \\
& -\sum_{i=1}^{k} \mathcal{T}_{m}^{r} f\left(\sqrt[p]{\left((k-1) m^{p}+1\right) x_{i}^{p}}\right), g(z) \\
& +(k-1)\left\|\mathcal{T}_{m}^{r} f\left(m \sqrt[p]{\sum_{i=1}^{k} x_{i}^{p}}\right)-\sum_{i=1}^{k} \mathcal{T}_{m}^{r} f\left(m x_{i}\right), g(z)\right\| \\
& \leq \alpha_{m}^{r} \prod_{i=1}^{k} h_{i}\left(\left((k-1) m^{p}+1\right) x^{p}, z\right)+(k-1) \alpha_{m}^{r} \prod_{i=1}^{k} h_{i}\left(m^{p} x^{p}, z\right) \\
& \leq \alpha_{m}^{r} \prod_{i=1}^{k} h_{i}\left(x^{p}, z\right)\left(\prod_{i=1}^{k} \lambda_{i}\left(\left((k-1) m^{p}+1\right)\right)+(k-1) \prod_{i=1}^{k} \lambda_{i}\left(m^{p}\right)\right) \\
& =\alpha_{m}^{r+1} \prod_{i=1}^{k} h_{i}\left(x^{p}, z\right)
\end{aligned}
$$

Thus, by induction, we have shown that (3.13) holds for every $x_{i}, z \in \mathbf{R}$, $n \in \mathbf{N}_{0}$, and $m \in \mathcal{U}$. Letting $n \rightarrow \infty$ in (3.13), we obtain the equality

$$
F_{m}\left(\sqrt[p]{\sum_{i=1}^{k} x_{i}^{p}}\right)=\sum_{i=1}^{k} F_{m}\left(x_{i}\right), \quad x_{i} \in \mathbf{R}, m \in \mathcal{U} .
$$


This implies that $F_{m}: \mathbf{R} \rightarrow Y$, defined in this way, is a solution of the equation

$$
F(x)=F\left(\sqrt[p]{\left((k-1) m^{p}+1\right) x^{p}}\right)-(k-1) F(m x), \quad x \in \mathbf{R}, m \in \mathcal{U} .
$$

Next, we will prove that each $p$-radical function $F: \mathbf{R} \rightarrow Y$ satisfying the inequality

$$
\|f(x)-F(x), g(z)\| \leq L \prod_{i=1}^{k} h_{i}\left(x^{p}, z\right), \quad x, z \in \mathbf{R}
$$

with some $L>0$, is equal to $F_{m}$ for each $m \in \mathcal{U}$. To this end, we fix $m_{0} \in \mathcal{U}$ and $F: \mathbf{R} \rightarrow Y$ satisfying (3.16). From (3.12), for each $x, z \in \mathbf{R}$, we get

$$
\begin{aligned}
\left\|F(x)-F_{m_{0}}(x), g(z)\right\| & \leq\|F(x)-f(x), g(z)\|+\left\|f(x)-F_{m_{0}}(x), g(z)\right\| \\
& \leq L \prod_{i=1}^{k} h_{i}\left(x^{p}, z\right)+\varepsilon_{m_{0}}^{*}(x, z) \\
& \leq L_{0} \prod_{i=1}^{k} h_{i}\left(x^{p}, z\right) \sum_{n=0}^{\infty} \alpha_{m_{0}}^{n}
\end{aligned}
$$

where $L_{0}:=\left(1-\alpha_{m_{0}}\right) L+\prod_{i=1}^{k-1} \lambda_{i}\left(m_{0}^{p}\right)>0$ and we exclude the case that $h_{i}\left(x^{p}, z\right) \equiv 0$ which is trivial. Observe that $F$ and $F_{m_{0}}$ are solutions to equation (3.15) for all $m \in \mathcal{U}$. Next, we show that, for each $j \in \mathbf{N}_{0}$, we have

$$
\left\|F(x)-F_{m_{0}}(x), g(z)\right\| \leq L_{0} \prod_{i=1}^{k} h_{i}\left(x^{p}, z\right) \sum_{n=j}^{\infty} \alpha_{m_{0}}^{n}, \quad x, z \in \mathbf{R} .
$$

The case $j=0$ is exactly (3.17). We fix $k \in \mathbf{N}$ and assume that (3.18) holds for $j=k$. Then, in view of (3.17), for each $x, z \in \mathbf{R}$, we get 


$$
\begin{aligned}
\left\|F(x)-F_{m_{0}}(x), g(z)\right\|= & \| F\left(\sqrt[p]{\left((k-1) m_{0}^{p}+1\right) x^{p}}\right)-(k-1) F\left(m_{0} x\right) \\
- & F_{m_{0}}\left(\sqrt[p]{\left((k-1) m_{0}^{p}+1\right) x^{p}}\right) \\
+ & (k-1) F_{m_{0}}\left(m_{0} x\right), g(z) \| \\
\leq & \| F\left(\sqrt[p]{\left((k-1) m_{0}^{p}+1\right) x^{p}}\right) \\
- & F_{m_{0}}\left(\sqrt[p]{\left((k-1) m_{0}^{p}+1\right) x^{p}}\right), g(z) \| \\
& +(k-1)\left\|F\left(m_{0} x\right)-F_{m_{0}}\left(m_{0} x\right), g(z)\right\| \\
\leq & L_{0} \prod_{i=1}^{k} h_{i}\left(\left((k-1) m_{0}^{p}+1\right) x^{p}, z\right) \sum_{n=k}^{\infty} \alpha_{m_{0}}^{n} \\
& +(k-1) L_{0} \prod_{i=1}^{k} h_{i}\left(m_{0}^{p} x^{p}, z\right) \sum_{n=k}^{\infty} \alpha_{m_{0}}^{n} \\
= & L_{0}\left(\prod_{i=1}^{k} h_{i}\left(\left((k-1) m_{0}^{p}+1\right) x^{p}, z\right)\right. \\
+ & \left.(k-1) \prod_{i=1}^{k} h_{i}\left(m_{0}^{p} x^{p}, z\right)\right) \sum_{n=k}^{\infty} \alpha_{m_{0}}^{n} \\
\leq & L_{0} \alpha_{m_{0}} \prod_{i=1}^{k} h_{i}\left(x^{p}, z\right) \sum_{n=k}^{\infty} \alpha_{m_{0}}^{n} \\
= & L_{0} \prod_{i=1}^{k} h_{i}\left(x^{p}, z\right) \sum_{n=k+1}^{\infty} \alpha_{m_{0}}^{n} .
\end{aligned}
$$

This shows that (3.18) holds for $j=k+1$. Now we can conclude that the inequality (3.18) holds for all $j \in \mathbf{N}_{0}$. Now, letting $j \rightarrow \infty$ in (3.18), we get

$$
F=F_{m_{0}} .
$$

Thus, we have also proved that $F_{m}=F_{m_{0}}$ for each $m \in \mathcal{U}$, which (in view of (3.12)) yields

$$
\left\|f(x)-F_{m_{0}}(x), g(z)\right\| \leq \frac{\prod_{i=1}^{k-1} \lambda_{i}\left(m^{p}\right) \prod_{i=1}^{k} h_{i}\left(x^{p}, z\right)}{1-\alpha_{m}}, x, z \in \mathbf{R}, m \in \mathcal{U}
$$
$F$.

This implies (3.4) with $F=F_{m_{0}}$ and (3.19) confirms the uniqueness of

In the following theorem, we investigate the stability of the equation (1.23) using a similar method of proof of Theorem 3.1.

Theorem 3.2. Let $h: \mathbf{R}^{2} \rightarrow \mathbf{R}_{+}$be a function such that

$$
\mathcal{U}:=\left\{n \in \mathbf{N}: \beta_{n}:=\lambda\left((k-1) n^{p}+1\right)+(k-1) \lambda\left(n^{p}\right)<1\right\} \neq \phi,
$$


where

$$
\lambda(n):=\inf \left\{t \in \mathbf{R}_{+}: h\left(n x^{p}, z\right) \leq t h\left(x^{p}, z\right), \quad x, z \in \mathbf{R}\right\}
$$

for all $n \in \mathbf{N}$. Assume that $f: \mathbf{R} \rightarrow Y$ satisfies the inequality

$$
\left\|f\left(\sqrt[p]{\sum_{i=1}^{k} x_{i}^{p}}\right)-\sum_{i=1}^{k} f\left(x_{i}\right), g(z)\right\| \leq \sum_{i=1^{k}} h\left(x_{i}^{p}, z\right)
$$

for all $x_{i}, z \in \mathbf{R}$ with $i=1,2, \ldots, k$, where $g: X \rightarrow Y$ be a surjective mapping.. Then there exists a unique p-radical function $F: \mathbf{R} \rightarrow Y$ such that

$$
\|f(x)-F(x), g(z)\| \leq \lambda_{0} h\left(x^{p}, z\right)
$$

for all $x, z \in \mathbf{R}$, where

$$
\lambda_{0}:=\inf _{n \in \mathcal{U}}\left\{\frac{1+(k-1) \lambda\left(n^{p}\right)}{1-\lambda\left((k-1) n^{p}+1\right)-(k-1) \lambda\left(n^{p}\right)}\right\} .
$$

Proof. For $i=1,2, \ldots,(k-1)$, replacing in inequality (3.23) $x_{i}$ with $m x$ and $x_{k}$ with $x$, where $x_{i} \in \mathbf{R}$ and $m \in \mathbf{N}$, we get

$$
\left\|f\left(\sqrt[p]{\left((k-1) m^{p}+1\right) x^{p}}\right)-(k-1) f(m x)-f(x), g(z)\right\| \leq\left(1+(k-1) \lambda\left(m^{p}\right)\right) h\left(x^{p}, z\right)
$$

for all $x, z \in \mathbf{R}$. For each $m \in \mathbf{N}$, we define

$$
\mathcal{T}_{m} \xi(x):=\xi\left(\sqrt[p]{\left((k-1) m^{p}+1\right) x^{p}}\right)-(k-1) \xi(m x), \quad \xi \in Y^{\mathbf{R}}, x \in \mathbf{R}
$$

$\Lambda_{m} \delta(x, z):=\delta\left(\sqrt[p]{\left((k-1) m^{p}+1\right) x^{p}}, z\right)+(k-1) \delta(m x, z), \quad \delta \in \mathbf{R}_{+}^{\mathbf{R} \times \mathbf{R}}, x, z \in \mathbf{R}$,

$$
\varepsilon_{m}(x, z):=\left(1+(k-1) \lambda\left(m^{p}\right)\right) h\left(x^{p}, z\right), \quad x, z \in \mathbf{R} .
$$

As in Theorem 3.1 we observe that (3.25) takes form 


$$
\left\|f(x)-\mathcal{T}_{m} f(x), g(z)\right\| \leq \varepsilon_{m}(x, z), \quad x, z \in \mathbf{R}
$$

and $\Lambda_{m}$ has the form described in (1.17) and (1.16) is valid for every $\xi, \mu \in$ $Y^{\mathbf{R}}, x, z \in \mathbf{R}$. It is not hard to show that

$$
\left(\Lambda_{m}^{n} \varepsilon_{m}\right)(x, z) \leq\left(1+(k-1) \lambda\left(m^{p}\right)\right) h\left(x^{p}, z\right)\left(\lambda\left((k-1) m^{p}+1\right)+(k-1) \lambda\left(m^{p}\right)\right)^{n}
$$

for all $x, z \in \mathbf{R}$ and $n \in \mathbf{N}_{0}$. Therefore

$$
\begin{aligned}
\varepsilon_{m}^{*}(x, z) & =\sum_{n=0}^{\infty}\left(\Lambda_{m}^{n} \varepsilon_{m}\right)(x, z) \\
& \leq \sum_{n=0}^{\infty}\left(1+(k-1) \lambda\left(m^{p}\right)\right) h\left(x^{p}, z\right) \beta_{m}^{n} \\
& =\frac{\left(1+(k-1) \lambda\left(m^{p}\right)\right) h\left(x^{p}, z\right)}{1-\beta_{m}}<\infty
\end{aligned}
$$

for all $x, z \in \mathbf{R}$ and $m \in \mathcal{U}$, where

$$
\beta_{m}=\left(\lambda\left((k-1) m^{p}+1\right)+(k-1) \lambda\left(m^{p}\right)\right) .
$$

Also the remaining reasonings are analogous as in the proof of Theorem 3.1.

The following theorem concerns the $\eta$-hyperstability of (1.23) in 2Banach spaces. Namely, We consider functions $f: \mathbf{R} \rightarrow Y$ fulfilling (1.23) approximately, i.e., satisfying the inequality

$$
\left\|f\left(\sqrt[p]{\sum_{i=1}^{k} x_{i}^{p}}\right)-\sum_{i=1}^{k} f\left(x_{i}\right), g(z)\right\| \leq \eta(x, y, z), \quad x, y, z \in \mathbf{R},
$$

with $\eta: \mathbf{R}^{k+1} \rightarrow \mathbf{R}_{+}$is a given mapping. Then we find a unique $p$-radical function $F: \mathbf{R} \rightarrow Y$ which is close to $f$. Then, under some additional assumptions on $\eta$, we prove that the conditional functional equation (1.23) is $\eta$-hyperstable in the class of functions $f: \mathbf{R} \rightarrow Y$, i.e., each $f: \mathbf{R} \rightarrow Y$ satisfying inequality (3.31), with such $\eta$, must fulfil equation (1.23).

Theorem 3.3. Let $h_{i}$ and $\mathcal{U}$ be as in Theorem 3.1. Assume that

$$
\left\{\begin{array}{l}
\lim _{n \rightarrow \infty} \prod_{i=1}^{k-1} \lambda_{i}(n)=0 \\
\lim _{n \rightarrow \infty} \prod_{i=1}^{k} \lambda_{i}(n)=0
\end{array}\right.
$$

Then every $f: \mathbf{R} \rightarrow Y$ satisfying (3.3) is a solution of (1.23). 
Proof. Suppose that $f: \mathbf{R} \rightarrow Y$ satisfies (3.3). Then, by Theorem 3.1, there exists a mapping $F: \mathbf{R} \rightarrow Y$ satisfies (1.23) and

$$
\|f(x)-F(x), g(z)\| \leq \lambda_{0} \prod_{i=1}^{k} h_{i}\left(x^{p}, z\right)
$$

for all $x, z \in \mathbf{R}$, where

$$
\lambda_{0}:=\inf _{n \in \mathcal{U}}\left\{\frac{\prod_{i=1}^{k-1} \lambda_{i}\left(n^{p}\right)}{1-\prod_{i=1}^{k} \lambda_{i}\left((k-1) n^{p}+1\right)-(k-1) \prod_{i=1}^{k} \lambda_{i}\left(n^{p}\right)}\right\} .
$$

Since, in view of (3.32), $\lambda_{0}=0$. This means that $f(x)=F(x)$ for all $x \in \mathbf{R}$, whence

$$
f\left(\sqrt[p]{\sum_{i=1}^{k} x_{i}^{p}}\right)=\sum_{i=1}^{k} f\left(x_{i}\right), \quad x_{i} \in \mathbf{R}
$$

which implies that $f$ satisfies the functional equation (1.23) on $\mathbf{R}$.

\section{Some particular cases}

According to above theorems, we derive some particular cases from our main results.

Corollary 4.1. For $i=1,2, \ldots, k$, let $h_{i}: \mathbf{R}^{2} \rightarrow(0, \infty)$ be as in Theorem 3.1 such that

$\lim _{n \rightarrow \infty} \inf \sup _{x, z \in \mathbf{R}} \frac{\left.\prod_{i=1}^{k} h_{i}\left((k-1) n^{p}+1\right) x^{p}, z\right)+\prod_{i=1}^{k} h_{i}\left(n^{p} x^{p}, z\right)}{\prod_{i=1}^{k} h_{i}\left(x^{p}, z\right)}=0, \quad x, z \in \mathbf{R}$.

Assume that $f: \mathbf{R} \rightarrow Y$ satisfies (1.23). Then there exist a unique $p$-radical function $F: \mathbf{R} \rightarrow Y$ and a unique constant $a \in \mathbf{R}_{+}$with

$$
\|f(x)-F(x), g(z)\| \leq a \prod_{i=1}^{k} h_{i}\left(x^{p}, z\right), \quad x, z \in \mathbf{R} .
$$


Proof. By the definition of $\lambda_{i}(n)$ in Theorem 3.1, we observe that

$$
\begin{aligned}
& \quad \prod_{i=1}^{k} \lambda_{i}\left((k-1) n^{p}+1\right)=\sup _{x, z \in \mathbf{R}} \frac{\left.\prod_{i=1}^{k} h_{i}\left((k-1) n^{p}+1\right) x^{p}, z\right)}{\prod_{i=1}^{k} h_{i}\left(x^{p}, z\right)} \\
& \leq \sup _{x, z \in \mathbf{R}} \frac{\left.\prod_{i=1}^{k} h_{i}\left((k-1) n^{p}+1\right) x^{p}, z\right)+\prod_{i=1}^{k} h_{i}\left(n^{p} x^{p}, z\right)}{\prod_{i=1}^{k} h_{i}\left(x^{p}, z\right)} \\
& \quad \text { and } \\
& \quad \prod_{i=1}^{k} \lambda_{i}\left(n^{p}\right)=\sup _{x, z \in \mathbf{R}} \frac{\prod_{i=1}^{k} h_{i}\left(n^{p} x^{p}, z\right)}{\prod_{i=1}^{k} h_{i}\left(x^{p}, z\right)} \\
& \leq \sup _{x, z \in \mathbf{R}} \frac{\left.\prod_{i=1}^{k} h_{i}\left((k-1) n^{p}+1\right) x^{p}, z\right)+\prod_{i=1}^{k} h_{i}\left(n^{p} x^{p}, z\right)}{\prod_{i=1}^{k} h_{i}\left(x^{p}, z\right)} .
\end{aligned}
$$

Moreover,

$(k-1) \prod_{i=1}^{k} \lambda_{i}\left(n^{p}\right) \leq(k-1) \sup _{x, z \in \mathbf{R}} \frac{\left.\prod_{i=1}^{k} h_{i}\left((k-1) n^{p}+1\right) x^{p}, z\right)+\prod_{i=1}^{k} h_{i}\left(n^{p} x^{p}, z\right)}{\prod_{i=1}^{k} h_{i}\left(x^{p}, z\right)}$

Combining inequalities (4.5) and (4.6), we get

$\prod_{i=1}^{k} \lambda_{i}\left((k-1) n^{p}+1\right)+(k-1) \prod_{i=1}^{k} \lambda_{i}\left(n^{p}\right)$

$\leq k \sup _{x, z \in \mathbf{R}} \frac{\left.\prod_{i=1}^{k} h_{i}\left((k-1) n^{p}+1\right) x^{p}, z\right)+\prod_{i=1}^{k} h_{i}\left(n^{p} x^{p}, z\right)}{\prod_{i=1}^{k} h_{i}\left(x^{p}, z\right)}$.

Write

$$
\omega_{n}:=\sup _{x, z \in \mathbf{R}} \frac{\left.\prod_{i=1}^{k} h_{i}\left((k-1) n^{p}+1\right) x^{p}, z\right)+\prod_{i=1}^{k} h_{i}\left(n^{p} x^{p}, z\right)}{\prod_{i=1}^{k} h_{i}\left(x^{p}, z\right)} .
$$

From (4.1), there is a subsequence $\left\{\omega_{n_{r}}\right\}$ of a sequence $\left\{\omega_{n}\right\}$ such that $\lim _{r \rightarrow \infty} \omega_{n_{r}}=0$, that is,

(4.4) $\lim _{r \rightarrow \infty} \sup _{x, z \in \mathbf{R}} \frac{\left.\prod_{i=1}^{k} h_{i}\left((k-1) n_{r}^{p}+1\right) x^{p}, z\right)+\prod_{i=1}^{k} h_{i}\left(n_{r}^{p} x^{p}, z\right)}{\prod_{i=1}^{k} h_{i}\left(x^{p}, z\right)}=0$.

From (4.6) and (4.7), we find that

$$
\lim _{r \rightarrow \infty} \prod_{i=1}^{k} \lambda_{i}\left((k-1) n_{r}^{p}+1\right)+(k-1) \prod_{i=1}^{k} \lambda_{i}\left(n_{r}^{p}\right)=0 .
$$

This implies that

$$
\lim _{r \rightarrow \infty} \prod_{i=1}^{k} \lambda_{i}\left(n_{r}^{p}\right)=0
$$


and hence

$\lim _{r \rightarrow \infty} \frac{\prod_{i=1}^{k-1} \lambda_{i}\left(n_{r}^{p}\right)}{1-\prod_{i=1}^{k} \lambda_{i}\left((k-1) n_{r}^{p}+1\right)-(k-1) \prod_{i=1}^{k} \lambda_{i}\left(n_{r}^{p}\right)}=\lim _{r \rightarrow \infty} \prod_{i=1}^{k-1} \lambda_{i}\left(n_{r}^{p}\right):=a$

which means that $\lambda_{0}$ defined in Theorem 3.1 is equal to $a$.

By a similar method, we can prove the following corollary where $a=1$.

Corollary 4.2. Let $h: \mathbf{R}^{2} \rightarrow(0, \infty)$ be as in Theorem 3.2 such that

(4.6) $\lim _{n \rightarrow \infty} \inf \sup _{x, z \in \mathbf{R}} \frac{h\left(\left((k-1) n^{p}+1\right) x^{p}, z\right)+h\left(n^{p} x^{p}, z\right)}{h\left(x^{p}, z\right)}=0, \quad x, z \in \mathbf{R}$.

Assume that $f: \mathbf{R} \rightarrow Y$ satisfies (1.23). Then there exist a unique $p$-radical function $F: \mathbf{R} \rightarrow Y$ such that

$$
\|f(x)-F(x), g(z)\| \leq h\left(x^{p}, z\right), \quad x, z \in \mathbf{R} .
$$

Corollary 4.3. Let $\theta \geq 0, r \geq 0$ and $q_{i} \in \mathbf{R}$ with $i=1,2, \ldots, k$ such that $\sum_{i=1}^{k} q_{i}<0$. Suppose that $f: \mathbf{R} \rightarrow Y$ such that $f(0)=0$ and satisfies the inequality

(4.8) $\left\|f\left(\sqrt[p]{\sum_{i=1}^{k} x_{i}^{p}}\right)-\sum_{i=1}^{k} f\left(x_{i}\right), g(z)\right\| \leq \theta \prod_{i=1}^{k}\left|x^{p}\right|^{q_{i}}|z|^{r}, \quad x_{i}, z \in \mathbf{R} \backslash\{0\}$,

where $p$ is odd, or $f$ is even function satisfies (4.11) where $p$ is even integer. Then $f$ satisfies (1.23) on $\mathbf{R}$.

Proof. The proof follows from Theorem 3.1 by defining $h_{i}: \mathbf{R}^{2} \rightarrow \mathbf{R}_{+}$ by $h_{i}\left(x_{i}^{p}, z\right)=\theta_{i}\left|x_{i}^{p}\right|^{q_{i}}|z|^{r}$ with $\theta_{i}, r \in \mathbf{R}_{+}$and $q_{i} \in \mathbf{R}$ such that $\prod_{i=1}^{k} \theta_{i}=\theta$ and $\sum_{i=1}^{k} q_{i}<0$.

For each $n \in \mathbf{N}$, we have

$$
\begin{aligned}
\lambda_{i}(n) & =\inf \left\{t \in \mathbf{R}_{+}: h_{i}\left(n x^{p}, z\right) \leq t h_{i}\left(x^{p}, z\right), \quad x, z \in \mathbf{R}\right\} \\
& =\inf \left\{t \in \mathbf{R}_{+}: \theta_{i}\left|n x^{p}\right|^{q_{i}} \leq t \theta_{i}\left|x^{p}\right|^{q_{i}}|z|^{r}, \quad x, z \in \mathbf{R} \backslash\{0\}\right\} \\
& =n^{q_{i}} .
\end{aligned}
$$

Clearly, we can find $n_{0} \in \mathbf{N}$ such that 


$$
\begin{aligned}
& \prod_{i=1}^{k} \lambda_{i}\left((k-1) n^{p}+1\right)+(k-1) \prod_{i=1}^{k} \lambda_{i}\left(n^{p}\right)=\left((k-1) n^{p}+1\right)^{\sum_{i=1}^{k} q_{i}} \\
& \quad+(k-1)\left(n^{p}\right)^{\sum_{i=1}^{k} q_{i}}<1, \quad n \geq n_{0} .
\end{aligned}
$$

According to Theorem 3.1, there exists a unique $p$-radical function $F$ : $\mathbf{R} \rightarrow Y$ such that

$$
\|f(x)-F(x), g(z)\| \leq \theta \lambda_{0}\left|x^{p}\right|^{\sum_{i=1}^{k} q_{i}}|z|^{r}
$$

for all $x, z \in \mathbf{R}$, where

$$
\lambda_{0}:=\inf _{n \geq n_{0}}\left\{\frac{\prod_{i=1}^{k-1} \lambda_{i}\left(n^{p}\right)}{1-\prod_{i=1}^{k} \lambda_{i}\left((k-1) n^{p}+1\right)-(k-1) \prod_{i=1}^{k} \lambda_{i}\left(n^{p}\right)}\right\} .
$$

On the other hand, Since $\sum_{i=1}^{k} q_{i}<0$, one of $q_{i}$ must be positive. Assume that $q_{k}>0$. Then

$$
\left\{\begin{array}{l}
\lim _{n \rightarrow \infty} \prod_{i=1}^{k-1} \lambda_{i}(n)=\lim _{n \rightarrow \infty} n^{\sum_{i=1}^{k-1} q_{i}}=0 \\
\lim _{n \rightarrow \infty} \prod_{i=1}^{k} \lambda_{i}(n)=\lim _{n \rightarrow \infty} n^{\sum_{i=1}^{k} q_{i}}=0 .
\end{array}\right.
$$

Thus by Theorem 3.3, we get the desired results.

The next corollary prove the hyperstability results for the inhomogeneous $p$-radical functional equation.

Corollary 4.4. Let $\theta, r, q_{i} \in \mathbf{R}$ such that $\theta \geq 0, r \geq 0$ and $\sum_{i=1}^{k} q_{i}<0$. Assume that $G: \mathbf{R}^{k} \rightarrow Y$ and $f: \mathbf{R} \rightarrow Y$ such that $f(0)=0$ satisfy the inequality

$$
\left\|f\left(\sqrt[p]{\sum_{i=1}^{k} x_{i}^{p}}\right)-\sum_{i=1}^{k} f\left(x_{i}\right)-G\left(x_{1}, x_{2}, \ldots, x_{k}\right), g(z)\right\| \leq \theta \prod_{i=1}^{k}\left|x^{p}\right|^{q_{i}}|z|^{r}
$$

for all $x_{i}, z \in \mathbf{R} \backslash\{0\}$ where $p$ is odd, or $f$ is even function satisfies (4.15) where $p$ is even integer. If the functional equation

$$
f\left(\sqrt[p]{\sum_{i=1}^{k} x_{i}^{p}}\right)=\sum_{i=1}^{k} f\left(x_{i}\right)+G\left(x_{1}, x_{2}, \ldots, x_{k}\right), \quad x_{1}, x_{2}, \ldots, x_{k} \in \mathbf{R} \backslash\{0\},
$$

has a solution $f_{0}: \mathbf{R} \rightarrow Y$, then $f$ is a solution to (4.16). 
Proof. From (4.15) we get that the function $K: \mathbf{R} \rightarrow Y$ defined by $K:=f-f_{0}$ satisfies (4.11). Consequently, Corollary 4.3 implies that $K$ is a solution to $p$-radical functional equation (1.23). Therefore,

$$
\begin{gathered}
f\left(\sqrt[p]{\sum_{i=1}^{k} x_{i}^{p}}\right)-\sum_{i=1}^{k} f\left(x_{i}\right)-G\left(x_{1}, x_{2}, \ldots, x_{k}\right) \\
=K\left(\sqrt[p]{\sum_{i=1}^{k} x_{i}^{p}}\right)+f_{0}\left(\sqrt[p]{\sum_{i=1}^{k} x_{i}^{p}}\right) \\
-\sum_{i=1}^{k} K\left(x_{i}\right)-\sum_{i=1}^{k} f_{0}\left(x_{i}\right)-G\left(x_{1}, x_{2}, \ldots, x_{k}\right) \\
=0
\end{gathered}
$$

for all $x_{1}, x_{2}, \ldots, x_{k} \in \mathbf{R} \backslash\{0\}$ which means $f$ is a solution to (4.16).

\section{References}

[1] L. Aiemsomboon and W. Sintunavarat, "On a new type of stability of a radical quadratic functional equation using Brzdęk's fixed point theorem", Acta mathematica hungarica, vol. 151, no. 1, pp. 35-46, Nov. 2016, doi: 10.1007/s10474-016-0666-2

[2] L. Aiemsomboon and W. Sintunavarat, "On generalized hyperstability of a general linear equation", Acta mathematica hungarica, vol. 149, no. 2, pp. 413-422, May 2016, doi: 10.1007/s 10474-016-0621-2

[3] Z. Alizadeh and A. G. Ghazanfari, "On the stability of a radical cubic functional equation in quasi- $\beta$-spaces", Journal of fixed point theory and applications, vol. 18, no. 4, pp. 843-853, Aug. 2016, doi: $10.1007 / \mathrm{s} 11784-016-0317-9$

[4] M. Almahalebi and A. Chahbi, "Approximate solution of P-radical functional equation in 2-Banach spaces," Acta mathematica scientia, vol. 39, no. 2, pp. 551-566, Mar. 2019, doi: $10.1007 / \mathrm{s} 10473-019-0218-2$

[5] T. Aoki, "On the stability of the linear transformation in Banach spaces”, Journal of the Mathematical Society of Japan, vol. 2, no. 1-2, pp. 64-66, Sep. 1950, doi: 10.2969/ jms j/ 00210064 
[6] J. Brzdęk, "A note on stability of additive mappings", in Stability of Mappings of Hyers-Ulam Type, T. M. Rassias and J. Tabor, Eds. Palm Harbor, FL: Hadronic Press, 1994, pp. 19-22.

[7] J. Brzdęk and K. Ciepliński, "A fixed point approach to the stability of functional equations in non-Archimedean metric spaces", Nonlinear analysis: theory, methods \& applications, vol. 74, no. 18, pp. 6861-6867, Dec. 2011, doi: 10.1016/ j.na.2011.06.050

[8] J. Brzdęk, "Stability of additivity and fixed point methods", Fixed point theory and applications, vol. 2013, no. 1, Art ID. 285, Nov. 2013, doi: 10.1186/1687-1812-2013-285

[9] J. Brzdesk "Hyperstability of the Cauchy equation on restricted domains," Acta mathematica hungarica, vol. 141, no. 1-2, pp. 58-67, Feb. 2013, doi: 10.1007/ s10474-013-0302-3

[10] J. Brzdęk, L. Cădariu, and K. Ciepliński, "Fixed Point Theory and the Ulam Stability", Journal of function spaces, vol. 2014, Art ID. 829419, 2014, doi: 10.1155/2014/829419

[11] J. Brzdęk, W. Fechner, M. S. Moslehian, and J. Sikorska, "Recent developments of the conditional stability of the homomorphism equation", Banach journal of mathematical analysis, vol. 9, no. 3, pp. 278-326, 2015, doi: 10.15352/ bjma/ 09-3-20

[12] J. Brzdęk, "3. Remark", 16th International Conference on Functional Equations and Inequalities, Będlewo, Poland, May 17-23, 2015, p. 196, Dec. 2015, doi: 10.1515/ aupcsm-2015-0012

[13] J. Brzdęk and K. Ciepliński, "On a fixed point theorem in 2-Banach spaces and some of its applications", Acta mathematica scientia, vol. 38, no. 2, pp. 377-390, Mar. 2018, doi: 10.1016/S0252-9602 (18) $30755-0$

[14] M. E. Gordji, H. Khodaei, A. Ebadian, and G. H. Kim, "Nearly radical quadratic functional equations in p-2-normed spaces", Abstract and applied analysis, vol. 2012 Art ID. 896032, 2012, doi: 10.1155/2012/ 896032

[15] M. Eshaghi Gordji and M. Parviz, "On the Hyers Ulam stability of the functional equation", Nonlinear functions analysis applications, vol.14, no. 3, pp. 413-420, 2009. [On line]. Available: https:// bit.ly/3pVAIDN

[16] S. Gähler, "2-metrische Räume und ihre topologische Struktur", Mathematische Nachrichten, vol. 26, no. 1-4, pp. 115-148, 1963, doi: 10.1002/ mana.19630260109 
[17] S. Gähler, "Linear 2-normiete Räumen", Mathematische Nachrichten, vol. 28, no. 1-2, pp. 1-43, 1964.

[18] Z. Gajda, "On stability of additive mappings", International journal mathematics sciences, vol. 14, no. 3, pp. 431-434, 1991, doi: $10.1155 /$ S016117129100056X

[19] D. H. Hyers, "On the stability of the linear functional equation", Proceedings of the National Academy of Sciences of the United States of America, vol. 27, no. 4, pp. 222-224, 1941, doi: 10.1073/ pnas.27.4.222

[20] H. Khodaei, M. E. Gordji, S. Kim, and Y. Cho, "Approximation of radical functional equations related to quadratic and quartic mappings", Journal of mathematical analysis and applications, vol. 395, no. 1, pp. 284-297, Nov. 2012, doi: 10.1016/ j.jmaa.2012.04.086

[21] S. Kim, Y. Cho, and M. E. Gordji, "On the generalized Hyers-UlamRassias stability problem of radical functional equations", Journal of inequalities and applications, Art ID. 186, Aug. 2012, doi: 10.1186/1029-242X-2012-186

[22] W. -G. Park, "Approximate additive mappings in 2-Banach spaces and related topics", Journal mathematics analysis applications, vol. 376, no. 1, pp. 193-202, Apr. 2011, doi: 10.1016/ j.jmaa.2010.10.004

[23] J. M. Rassias, "On approximation of approximately linear mappings by linear mappings", Journal functions analysis, vol. 46, no. 1, pp. 126-130, Mar. 1982, doi: 10.1016/0022-1236(82)90048-9

[24] J. M. Rassias, "Solution of a problem of Ulam", Journal of approximation theory, vol. 57, no. 3, pp. 268-273, Jun. 1989, doi: 10.1016/0021-9045(89)90041-5

[25] T. Rassias, "On a modified Hyers-Ulam sequence", Journal mathematics analysis applications, vol. 158, no. 1, pp. 106-113, Jun. 1991, doi: 10.1016/0022-247X(91)90270-A

[26] S. M. Ulam, Problems in modern mathematics. New York, NY: J. Wiley, 1964. 